\title{
Chapter 14 \\ MRI Technology for Behavioral \\ and Cognitive Studies in Macaques In Vivo
}

\author{
Yong Zhu and Paul A. Garber
}

\subsection{Introduction}

Primate behavior, especially social behavior, might seem a strange place to begin in trying to advance our understanding of the brain or human mind (Opstal 1996; Critchley and Harrion 2013). Primate brains differ in structural details, proportion among functional areas, as well as in overall size, implying specific adaptations to the challenges posed by particular ecological and social environments across that species' evolutionary history. Given that brains require a disproportionate amount of nutrients and energy relative to other body organs (Aiello and Wheeler 1995), an understanding of the functional implications of evolutionary changes in brain organization is critical for evaluating relationships between cognition, decision-making, and behavior (Rilling 2006) (Fig. 14.1).

The recent decade has seen an explosion of interest and information about brain connectivity and functions over a wide range of spatial scales, including macroscopic, microscopic, and mesoscopic levels (Essen et al. 2016). Noninvasive imaging studies with magnetic resonance imaging (MRI) technologies have been used for over 30 years (Vanduffel 2018) and have made fundamental contributions to our understanding of animal behavior and cognition, especially in our understanding of the relationships between brain structures and brain function. For example, the brain's default mode network (DMN) consists of discrete, bilateral, and symmetrical cortical areas in the medial and lateral parietal, medial prefrontal, and medial and lateral temporal cortices of the human, nonhuman primate, cat, and rodent brains

Y. Zhu $(\bowtie)$

High Magnetic Field Laboratory, Chinese Academy of Sciences, Hefei, China

School of Life Sciences, Hefei Normal University, Hefei, Anhui, China

P. A. Garber

Department of Anthropology, Program in Ecology, Evolution, and Conservation Biology,

University of Illinois, Urbana, IL, USA

e-mail: p-garber@illinois.edu 


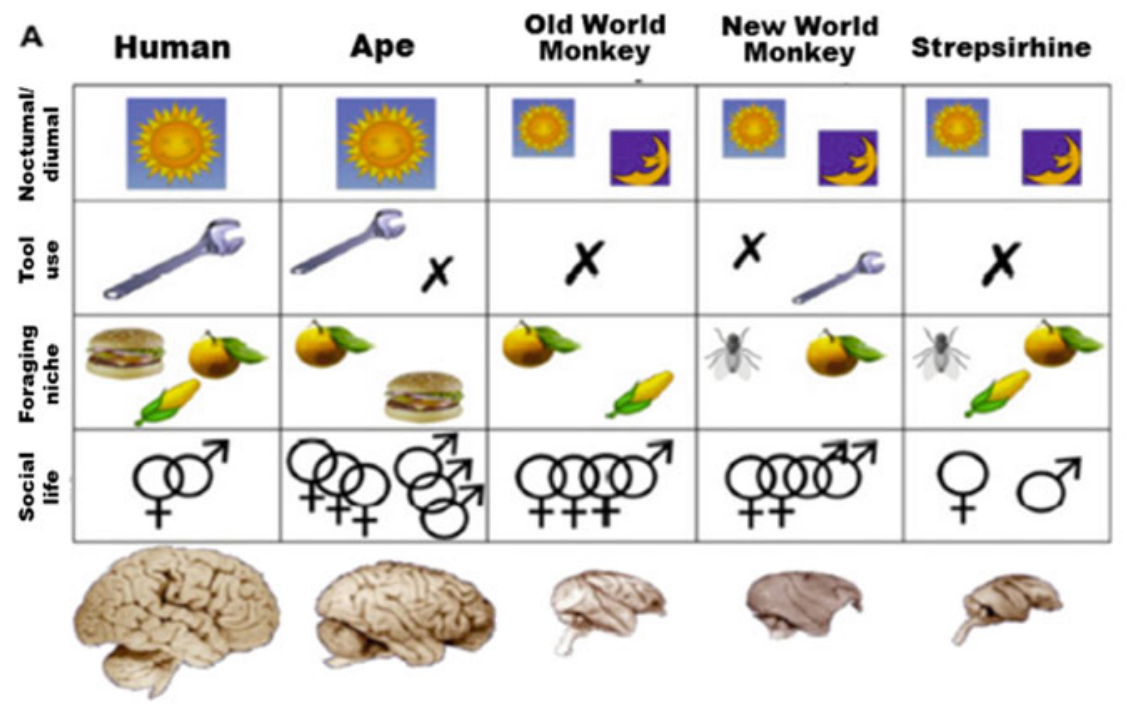

\section{B (Post-mortem) Samples \\ MRI \\ Imaging Statistical Relation to data comparison behavior}

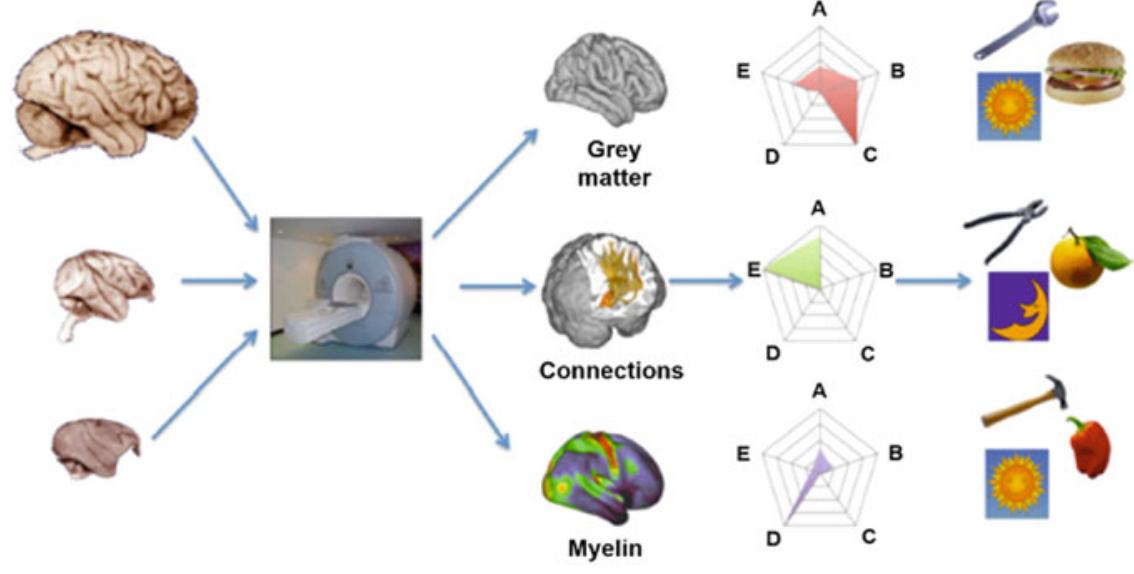

Fig. 14.1 Analyzing the relationship between brains and behavior (Mars et al. 2014). (a) In a multivariate comparative approach, each brain is viewed as a unique combination of variables, including whether the animal is active during night or day; whether it uses tools regularly, occasionally, or not at all; what its diet is; or how complex its social life is. By using a wholebrain and multivariate approach, it is possible for us to investigate how differences in specific aspects of brain organization are related to different ecological and social variables. (b) MRI of whole-brain (postmortem) samples allows a number of measures to be collected, for which comparative analysis techniques have now been developed and validated 
(Raichle 2015). The DMN consistently decreases its activity when compared with the activity of other brain areas during relaxed states. Using fMRI in macaques, researchers have detected that a cortical network, activated during shifts in cognitive activity, largely overlapped with the DMN and therefore have proposed that cognitive shifting in primates generally recruits activity in DMN regions (Arsenault et al. 2018). Prior to 1991, it was virtually impossible to map brain activation rapidly and noninvasively with full brain coverage and relatively high spatial and temporal resolution (Bandettini 2009). The use of fMRI along with positron emission tomography (PET), has revolutionized cognitive neuroscience (Logothetis 2008). Using these noninvasive techniques, we can ask research questions such as the relationship between brain and behavior in primates, which could not have been studied otherwise.

Nonhuman primates (NHPs), especially macaques, have been used traditionally as a model for studying many aspects of human behavior, health, and biology. This includes social structure, social behavior, and social cognition. In this chapter, we describe recent advances in MRI technology (including the state-of-the-art high field MRI), review several fMRI and PET studies on macaques, and indicate how these studies have contributed new insights into an understanding of nonhuman and human primate cognition and behavior.

\subsection{Magnetic Resonance Imaging (MRI)}

\subsubsection{Background of MRI}

MRI is a painless, noninvasive tool commonly used to diagnose disease progression, injury, or other ailments. Since its discovery in 1945, nuclear magnetic resonance (NMRI) has been used extensively as an analytic tool in chemistry and physics. In the early 1970s, interest in localized tissue measurements and the realization that highly accurate internal images could be obtained expanded the potential applications of NMRI in medical research. By the 1980s, the quality of NMRI in humans had been improved to the point that the radiological community considered MRI as the next high technology imaging modality.

Today, we refer to "NMRI" as MRI. The use of word "nuclear" in the acronym was dropped to avoid a negative association with the potential exposure to nuclear radiation. Its working principle is based on the fact that certain atomic nuclei are able to absorb and emit radio-frequency energy when placed in an external magnetic field. In both its clinical application and in MRI research, hydrogen atoms are most often used to generate a detectable radio-frequency signal that is received by antennas in close proximity to the anatomical structure being examined. Hydrogen atoms are naturally abundant, particularly in water and fat. For this reason, most MRI scans essentially map the location of water and fat in the body. Pulses of radio waves excite the nuclear spin energy transition, and magnetic field gradients localize the signal in space. By varying the parameters of the pulse sequence, different contrasts may be generated between tissues based on the relaxation properties of the hydrogen atoms. 


\subsubsection{The Advantages of MRI}

Compared with other medical imaging techniques such as X-ray imaging, ultrasonic imaging, and computed tomography (CT), MRI is a painless and noninvasive method to view human or animal tissue and obtain anatomical and functional diagnostic information. While the hazards of X-rays are now well-controlled in most medical contexts, MRI may still be seen as a better choice than CT. MRI scanners are designed to visualize non-bony parts or "soft tissue" areas such as muscles, ligaments, and tendons. In particular, the brain, spinal cord, and nerves are seen much more clearly with MRI than with regular X-rays and CT scans.

What are the advantages of an MRI scan? To summarize, there are several as follows:

1. High accuracy

In clinical diagnostics and studies, MRI is used as the preferred medical examination tool owing to its high accuracy in the detection of serious ailments such as tumors and cancers. In research, it can be used in structural and functional connectivity studies. In addition, the MRI scan parameters can be adjusted for a more comprehensive view of the area of interest.

2. Less confining

An MRI scanner has a design that makes it less confining for the patient or research subject. It is possible to assign tasks to the subject in the scanner, for example, evaluate light or sound stimulation, and this facilitates directly linking behavior and functional anatomy.

3. Noninvasive

MRI does not involve X-rays or the use of ionizing radiation, distinguishing it from CT or CAT scans. This removes all ethical concerns regarding harm that could be done to patients or study subject. This permits the ethical use of fMRI research studies because normal subjects face no risk of harm or injury.

With these four main advantages, MRI represents a powerful tool well suited for studying in vivo behavioral responses, especially functional studies that link neuroanatomy and cognitive behavior in primates.

\subsubsection{State of the Art at High Field MRI}

As far as MRI is concerned, the need to achieve the highest possible magnetic field in clinical settings has been a major motivation for scientists and engineers to improve the imaging technology. As a result, MRI scanners have evolved from the first generation $(0.5$ Tesla or $0.5 \mathrm{~T})$ to the conventional (1.0-1.5 $\mathrm{T})$ scanner and then to the high field (3 T) scanners in clinical applications. Most recently, ultra-high field MRI scanners ranging from 7 to $11.7 \mathrm{~T}$ have been designed. Generally speaking, the higher the magnetic field, the better the resolution of the images.

Using MRI, a higher magnetic field intensity leads to a higher signal-to-noise ratio (SNR), resulting in a higher image resolution. However, higher magnetic field 
intensity can lead to a smaller caliber machine (the diameter of the machine that allows the animal to be scanned) meaning that animals larger than macaques (e.g., gorilla) could not be examined. For example, the $3 \mathrm{~T}$ MRI scanner in clinical applications usually has a $70 \mathrm{~cm}$ caliber, suited for human or gorilla body scans. The 9.4 T MRI scanner with a caliber ranging from 30 to $40 \mathrm{~cm}$ is suitable for NHPs ranging in size from a mouse lemur to a macaque. The $9.4 \mathrm{~T}$ MRI scanner obtains enhanced image resolution compared to the $3 \mathrm{~T}$ MRI scanner. Therefore, considering the image resolution, a 9.4 T MRI scanner is the most suitable for studying mammals ranging from rodents to most species of NHPs.

There are many 9.4 T MRI scanners currently in use. For example, a 9.4 T MRI scanner with a $40 \mathrm{~cm}$ diameter bore (Agilent, US) is operated at the High Magnetic Field Laboratory (HMFL), Chinese Academy of Sciences, Hefei. This scanner is dedicated to the study of larger mammals (e.g., dog, sheep, and macaque). Another $9.4 \mathrm{~T} / 30 \mathrm{~cm}$ MRI scanner (Bruker, Germany) is operated at the Shanghai Institute of Biological Sciences of the Chinese Academy of Sciences. This scanner focuses on scientific research requiring a high magnetic field. At the University of Chicago, Chicago, Illinois, a $9.4 \mathrm{~T} / 30 \mathrm{~cm}$ MRI facility is used for basic science research within the Department of Radiology. The goal is to advance state-of-the-art animal imaging technology at the anatomical and functional level (https://mris.uchicago.edu/). Many experimental studies of NHPs that focus on research questions including brain development, brain structural changes, and functional studies of different brain regions have been conducted using other types of 9.4 T MRI scanners such as the $9.4 \mathrm{~T} / 35 \mathrm{~cm}$ scanner in Germany (Goebel et al. 2009) and France (Même et al. 2015) and the $9.4 \mathrm{~T} / 39 \mathrm{~cm}$ scanner at University College London Centre for Advanced Biomedical Imaging, London (Ramasawmy et al. 2016).

Some primate studies have been carried out on $1.5 \mathrm{~T}$ or $3 \mathrm{~T}$ scanners (Nelissen and Vanduffel 2017), with a sub-optimal resolution in the range of 2-3 $\mathrm{mm}$. In vivo studies of structural brain imaging at $7 \mathrm{~T}$ have been reported in the rhesus macaque (Macaca mulatta), with resolution from 0.3 to $0.5 \mathrm{~mm}$ (Zitella et al. 2015). A much finer image resolution (under $100 \mu \mathrm{m}$ ) is needed to pick up minor changes, such as in brain aging that would be required to examine questions of ontogenetic changes in social behavior related to aging. However, only a limited number of primate studies have used 9.4 T MRI scanners, which would generate in vivo data with a resolution on the order of $0.1 \mathrm{~mm}$. This level of resolution is needed to examine questions related to the substructure of the hippocampus. Because dynamic brain alterations can be detected on a much finer scale, high field MRI greatly facilitates studies integrating primate brain structure and behavioral change.

\subsection{In Vivo MRI Study in Macaques}

Investigations into the structure and function of the NHP brain have significantly contributed to our overall understanding of cognition. 


\subsubsection{Structural MRI in Brain Imaging Study}

To understand the anatomical localization and functional activity in different cortical areas, it is necessary to obtain an accurate map of neural architectonic areas. In most MRI studies, primates are anesthetized when scanned. The latest research, published in the journal NeuroImage, has created an anatomical MRI brain template derived from 31 rhesus macaques (the macaques were juveniles and adults between 3.2 and 13.2 years old). The template also includes tissue maps, surfaces, and transformation scripts to assist in data analysis (Seidlitz et al. 2018). These data can be used to determine the variance of cortical topographies in the same individual over time and also compare cortical differences between infants, juveniles, and adults. This can be used as a framework for examining correlational relationships between changes in behavior and behavioral and brain development.

Unlike morphological studies using postmortem tissue samples, MRI allows the noninvasive, in vivo assessment of many different brain parameters including the topography and volume of gray and white matter in brain structures. At the level of the cerebral hemisphere, gray matter is mainly distributed in the periphery (cortex) while the white matter is located deep within the cortex.

Phillips and Sherwood (2008) described growth patterns in total brain volume, cortical gray and white matter volume, frontal lobe gray and white matter volume, and corpus callosum area in 29 brown capuchin monkeys (Sapajus nigritus, formerly Cebus apella) ranging in age from 4 days to 20 years. Of the total subjects, 12 were adults ( $\geq 5$ years) and 17 were juveniles (between 4 days to 5 years). The results revealed that nonlinear age-related changes in total brain volume, cortical white matter volume, and frontal white matter volume occur from birth to 5 years of age (subadult period of development). The implications of these results is the rapid increase of frontal lobe white matter during the first few years of life corresponds with opportunities for social learning and acquiring technical skills related to object manipulation, prey search, possibly tool use, and other complex foraging behaviors (Phillips and Sherwood 2008). Similarly, Wisco et al. (2008) studied the age-related white and gray matter volume changes in eight young adult (5-12 years), six middleaged adult (16-19 years) and eight old adult (24-30 years) rhesus macaques. The results found an overall decrease in the total forebrain $(5.01 \%)$, forebrain parenchyma $(5.24 \%)$, forebrain white matter $(11.53 \%)$, forebrain gray matter $(2.08 \%)$, caudate nucleus $(11.79 \%)$, and globus pallidus $(18.26 \%)$ with increasing age. Corresponding behavioral data for five of the younger, five of the middle-aged, and seven of the old adults on the delayed non-matching to sample (DNMS) task, the delayed recognition span task (DRST), and the cognitive impairment index (CII) found no correlation between these cognitive measures and ROI volume changes.

In a recent study, Scott et al. (2016) longitudinally assessed normative brain growth patterns using MRI in rhesus macaques. Cohort A consisted of 24 individuals (12 males, 12 females) and cohort B of 21 individuals (11 male, 10 female). They scanned the macaques at 1, 4, 8, 13, 26, 39, and 52 weeks of age. Cohort A had additional scans at 156 weeks (3 years) and 260 weeks (5 years) (Fig. 14.2). The 


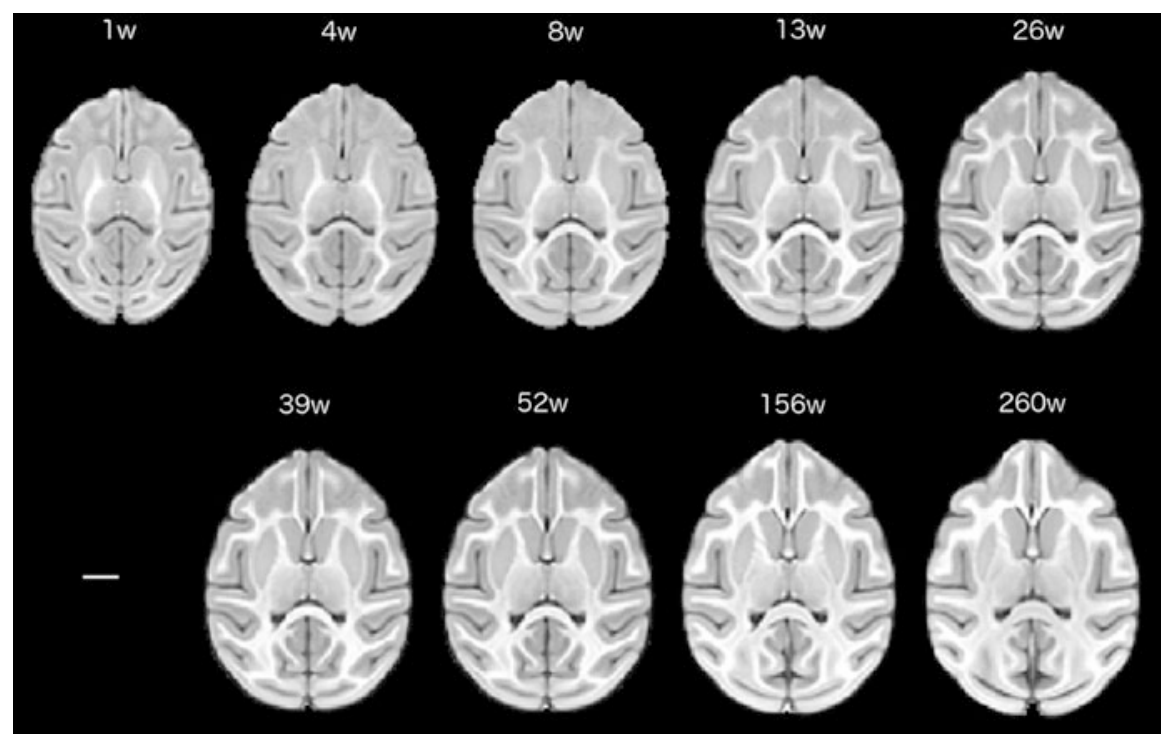

Fig. 14.2 Brain MRI at each study time point (Scott et al. 2016). Age-specific, horizontally oriented templates are shown for each study time point. Each template represents an average brain image constructed from individual subject scans. Images display enhanced signal-to-noise and optimal shape characteristics relative to individual scans (scale bar represents $1 \mathrm{~cm}$ )

results showed that total brain volume at 1 week was approximately $64 \%$ of that of the adult. Brain volume was larger in male rhesus macaques compared to females. While brain volume generally increased between any two imaging time points, there was a transient plateau of brain growth between 26 and 39 weeks in both males and females (Scott et al. 2016). This study serves as a starting point for more extensive analyses into the relationship between structural development of the brain and behavioral development of the rhesus macaques. The image database is available to behavioral scientists for addressing additional questions examining the relationship between changes in behavior and changes in neural development, including the emergence of sex and species typical behavior.

To evaluate hippocampal development in rhesus macaques, Hunsaker et al. (2014) obtained longitudinal structural MRI scans at 9 time points between 1 week and 260 weeks ( 5 years) of age in 24 rhesus macaques (12 males, 12 females) (Fig. 14.3). The results showed that the hippocampus reached $50 \%$ of its adult volume by 13 weeks of age and full adult volume by 52 weeks in both males and females. The hippocampus appears to be slightly larger at 3 years than at 5 years of age, and damage to the hippocampus deficit can result in permanent changes in behavior such as learning and memory, as well as neurocognitive function. Male rhesus macaques have a 5\% larger hippocampi than females from 8 weeks of age onward. Neuroimaging studies in rhesus macaques can provide critical information about the relationships between MRI volumetric changes and behavior during individual development. 


\section{Week}
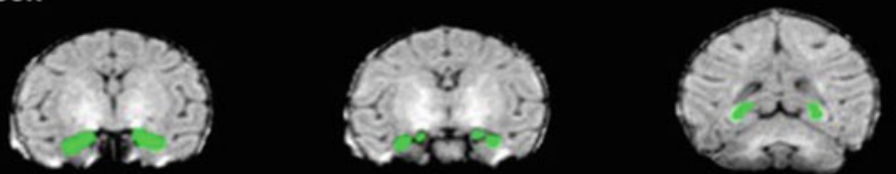

\section{Weeks}
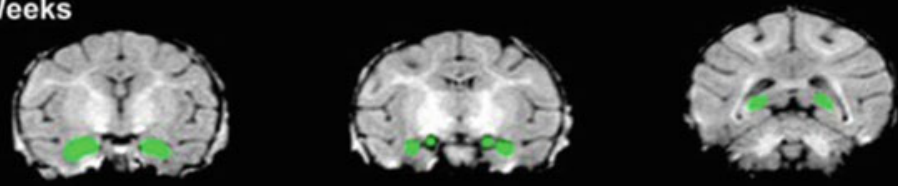

\section{Weeks}
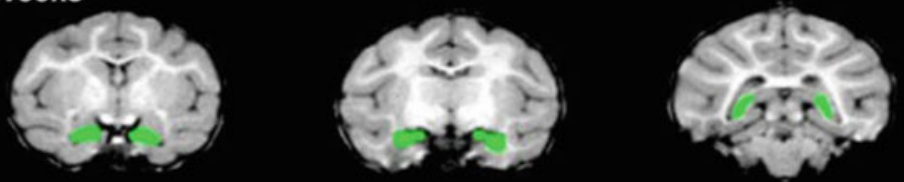

\section{Weeks}
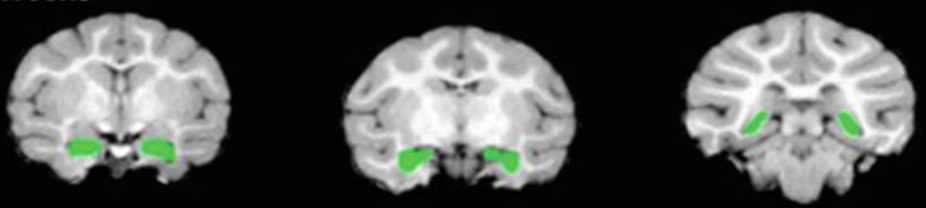

\section{Weeks}
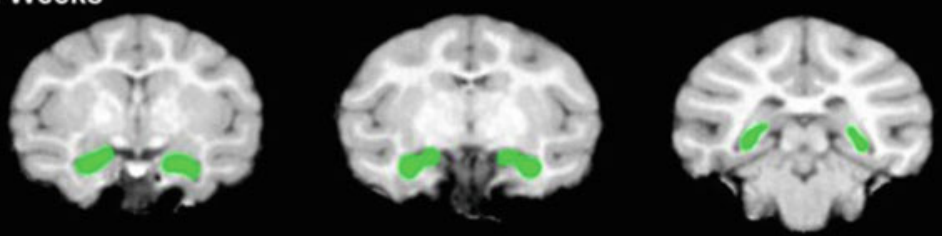

\section{Weeks}
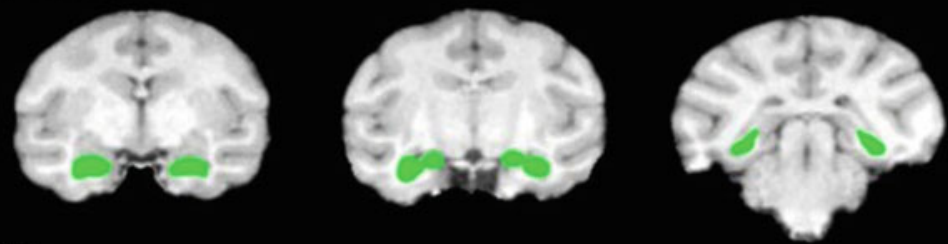

$1 \mathrm{~cm}$

Fig. 14.3 MRI for hippocampal tracings at different ages (Hunsaker et al. 2014). Sample hippocampal tracings from a single male rhesus macaque. Shown are scans at 1 week, 4 week, 26 weeks, 39 weeks, and 260 weeks of age. Note the difference in white matter at the different ages. All scans are shown at the same scale for direct visual comparison. Note the general shape of the traced hippocampus and size relative to the rest of brain at different ages (scale bar $=1 \mathrm{~cm}$ ) 
Liu et al. (2015) examined brain development in 14 male rhesus macaques from 6 to 16 months of age. The results showed rapid growth $(6.21 \%)$ in brain volume during early development between 6 and 10 months of age compared to a $2.81 \%$ growth rate between 10 and 16 months of age. Early expansion is mainly the result of a significant increase in white matter volume, while the later decline can be partly explained by a significant decrease of gray matter volume after 10 months of age. Compared with macaque brain development, human brain volume increases by $100 \%$ during the first year of life and then maintains cubic growth into adolescence. The pattern of human brain growth differs from the rhesus macaque in that humans have a less mature brain at birth (approximately $25 \%$ of adult size) and a longer period of early rapid brain growth during the first year of life (Phillips and Sherwood 2008).

\subsubsection{Functional MRI in Brain Imaging Study}

Functional magnetic resonance imaging (fMRI) has been used extensively in comparative sensory and cognitive experiments associated with brain activation mapping in NHPs and humans (Logothetis et al. 1999; Nakahara et al. 2002; Mantini et al. 2012). Moreover given that the subject is alert, one can study relationships between behavior, decision-making, and neural activity in real time. What exactly does fMRI tell us? We know that its signals arise from changes in local brain hemodynamics that, in turn, result in alterations in neuronal activity. However, exactly how neuronal activity, hemodynamics, fMRI signals, and decision-making are related is still unclear. It has been assumed that the fMRI signal is proportional to the local average neuronal activity, but many factors can influence this relationship (Heeger and Ress 2002).

For comparative studies of human and nonhuman primates, subjects are exposed to the same set of images as their brains are scanned using fMRI. The time course of fMRI activity during viewing is extracted from "seed" regions in human participants and correlated with the fMRI signal in NHP, or vice versa, with an adjustment made for interspecific differences in vascular hemodynamics. After statistical thresholding, which is a method of image processing, the resulting maps for each species show areas that are stimulated and potentially homologous with the targeted seed region in the other species (Wager and Yarkoni 2012) (Fig. 14.4). fMRI has been used to study the primate visual system (Logothetis et al. 1999; Nakahara et al. 2002; Russ and Leopold 2015), the auditory system (Mantini et al. 2012; Ortiz-Rios et al. 2015), and the motor system (Bauman et al. 2013), again principally in macaques. We present the results of some of these studies below.

Ortiz-Rios et al. (2015) investigated how species-specific vocalizations are represented in auditory and auditory-related regions of the brain using fMRI in rhesus macaques. The results indicated that these vocalizations preferentially activated the auditory ventral stream and in particular areas of the anterolateral belt and parabelt. 


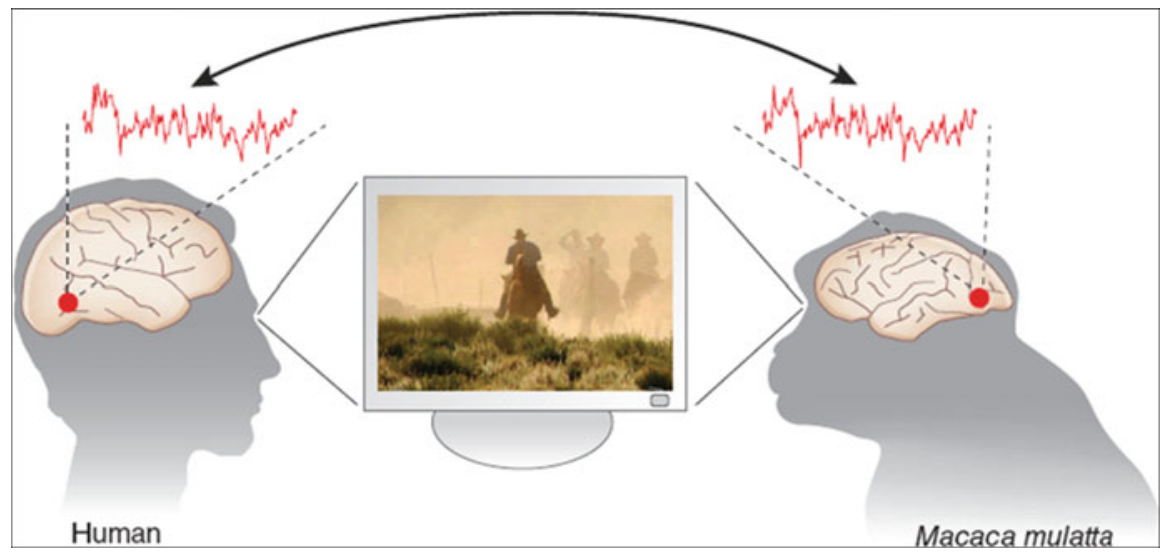

Fig. 14.4 Humans and monkeys watch the same film as their brains are scanned with fMRI (Wager and Yarjoni 2012). The time course of fMRI activity during viewing is extracted from "seed" regions in human participants and correlated with the fMRI signal in monkeys, and vice versa, with an adjustment made for interspecific differences in vascular hemodynamics

A major limitation of fMRI studies of nonhuman primates is the difficulty of training monkeys and apes to remain calm when placed in the restraining devices required to limit movement during scanning. Srihasam et al. (2010) developed a technique for holding subjects' heads motionless during scanning using a customfitted plastic helmet, chin strap, and mild suction supplied by a vacuum blower. This vacuum helmet method appeared to have few adverse effects on subject physical health (although the monkeys were stressed) even after repeated use for several months.

\subsection{Conclusion}

Studies of the brain of nonhuman primates are vital for understanding aggressive and cooperative interactions (Rilling 2014). Noninvasive imaging technologies, such as fMRI, are an important research tool that provide data that field primatologists can use to better understand how age, sex, and species differences in neural development, social experience, and neurohormones affect behavior and cognitive processes. For example, differences in patterns and rates of brain growth may help explain taxonomic differences in maternal pre- and postnatal offspring investment; differences in the manner in which species encode, store, and integrate temporal, spatial, and quantitative information in deciding where to feed; and species-specific differences in cooperative behavior. For example, oxytocin, a neurohormone synthesized in the hypothalamus, can increase cooperative behavior and reduce fear of cheaters in humans by stimulating brain regions that associate cooperative interactions with feelings of pleasure or reward (Rilling 2014). In the case of wild 
chimpanzees, Wittig et al. (2014) found that during periods of food sharing, levels of urinary oxytocin concentration increased significantly compared to periods when chimpanzees fed alone. Moreover this effect was evident in chimpanzees who shared food with close partners and in chimpanzees who shared food with nonpartners (Wittig et al. 2014). Similarly, Crockford et al. (2013) found that oxytocin levels in wild chimpanzees increased equally when individuals groomed kin or nonkin. Thus, there exists an important neurohormonal mechanism for promoting social cooperation between both related and nonrelated group members. In the case of common marmosets (Callithrix jacchus), a small-bodied New World monkey, Finkenwirth et al. (2016) examined relationships between nonmaternal infant caregiving and urinary oxytocin levels. In this species, both male and female helpers were found to exhibit significantly higher levels of urinary oxytocin when caring for offspring than during periods when not providing infant care. Finally, different species of macaques vary in dominance style from highly aggressive to highly affiliative. Although early experience may contribute to the ways in which individuals interact with others, a study by Rosenblum et al. (2002) reported that bonnet macaques (M. radiata), which are highly affiliative, were characterized by higher levels of oxytocin than pig-tailed macaques ( $M$. nemistrina) which are highly aggressive. Combined, these studies highlight the integrated role of neurohormones, neuroreceptors, and social experience in understanding primate social interactions and behavior.

Neurodevelopmental research is expected to expand in the future, offering new insights and understanding into links between neural anatomy and behavior, both within and between species. For example, neurodevelopmental studies may allow us to understand how the brain neuronal functions organize and mature during different stages of development. This will allow researchers to address questions linking behavior, ontogeny, and social cognition in primates.

Acknowledgments We would like to thank Dr. K. Zhong, H. Y. Yang, J. Zhang, Q. J. Zhu, and H. Y. Tong at the High Magnetic Field Laboratory, the Chinese Academy of Sciences. We also thank Dr. L. X. Sun at Central Washington University for his many helpful comments and suggestions on this chapter. PAG wishes to thank Chrissie, Sara, and Jenni for their love and support. This study was supported by the National Natural Science Foundation of China (31501866) and the Science and Technology Innovation Program of the Ministry of Science and Technology of the People's Republic of China (2014BAL03B00).

\section{References}

Aiello LC, Wheeler P (1995) The expensive-tissue hypothesis: the brain and the digestive system in human and primate evolution. Curr Anthropol 36:199-221

Arsenault JT, Caspari N, Vandenberghe R, Vanduffel W (2018) Attention shifts recruit the monkey default mode network. J Neurosci 38:1202-1217

Bandettini PA (2009) Functional MRI limitations and aspirations. In: Kraft E, Gulyás B, Pöppel E (eds) Neural correlates of thinking. Springer-Verlag, Berlin, pp 15-38

Bauman MD, Losif AM, Ashwood P, Braunschweig D, Lee A, Schumann CM, Water JV, Amaral DG (2013) Maternal antibodies from mothers of children with autism alter brain growth and social behavior development in the rhesus monkey. Transl Psychiatry 3(7):e278 
Critchley HD, Harrison NA (2013) Visceral influences on brain and behavior. Neuron 77:624-638

Crockford C, Wittig RM, Langergraber K, Ziegler TE, Zuberbuhler K, Deschner T (2013) Urinary oxytocin and social bonding in related and unrelated wild chimpanzees. Proc Biol Sci 280:20122765

Essen DCV, Donahue C, Dierker DL, Glasser MF (2016) Parcellations and connectivity patterns in human and macaque cerebral cortex. In: Kennedy H, Essen DCV, Christen Y (eds) Micro-, meso- and macro-connectomics of the brain. Springer International, Cham, pp 89-106

Finkenwirth C, Martins E, Deschner T, Burkart JM (2016) Oxytocin is associated with infant-care behavior and motivation in cooperatively breeding marmoset monkeys. Horm Behav 80:10-18

Goebel H, Spehl T, Paul D, Markl M, Harloff A (2009) Histopathological correlation and feasibility of atherosclerotic carotid lesion classification using $\mathrm{T}_{2} *$ weighted imaging at 9.4t MRI. Virchows Archiv 455:50-51

Heeger DJ, Ress D (2002) What does fMRI tell us about neuronal activity? Nat Rev Neurosci 3:142-151

Hunsaker MR, Scott JA, Bauman MD, Amaral DG (2014) Postnatal development of the hippocampus in the Rhesus macaque (Macaca mulatta): a longitudinal magnetic resonance imaging study. Hippocampus 24:794-807

Liu CR, Tian XG, Liu HL, Mo Y, Bai Y, Zhao XD, Ma YY, Wang JH (2015) Rhesus monkey brain development during late infancy and the effect of phencyclidine: a longitudinal MRI and DTI study. NeuroImage 107:65-75

Logothetis NK (2008) What we can do and what we cannot do with fMRI. Nature 453:869-878

Logothetis NK, Guggenberger HG, Peled S, Pauls J (1999) Functional imaging of the monkey brain. Nat Neurosci 2:555-562

Mantini D, Hasson U, Betti V, Perrucci MG, Romani GL, Corbetta M, Orban GA, Vanduffel W (2012) Inter-species activity correlations reveal functional correspondences between monkey and human brain areas. Nat Methods 9:277-282

Mars RB, Neubert FX, Verhagen L, Sallet J, Miller KL, Dunbar RIM, Barton RA (2014) Primate comparative neuroscience using magnetic resonance imaging: promises and challenges. Front Neurosci 8:298

Même S, Joudiou N, Yousfi N, Szeremeta F, Lopes-Pereira P, Beloeil JC, Herault Y, Meme W (2015) In vivo 9.4T MRI and ${ }^{1} \mathrm{H}$ MRS for evaluation of brain structural and metabolic changes in the Ts65Dn mouse model for Down syndrome. World J Neurosci 4:152-163

Nakahara K, Hayashi T, Konishi S, Miyashita Y (2002) Functional MRI of macaque monkeys performing a cognitive set-shifting task. Science 295:1532-1536

Nelissen K, Vanduffel W (2017) Action categorization in rhesus monkeys: discrimination of grasping from non-grasping manual motor acts. Sci Rep 7:15094

Opstal VA (1996) Dynamic patterns: the self-organization of brain and behavior. Complexity 2:253-254

Ortiz-Rios M, Kuśmierek P, Dewitt I, Archakov D, Azevedo FAC, Sams M, Jääskeläinen IP, Keliris GA, Rauschecker JP (2015) Functional MRI of the vocalization-processing network in the macaque brain. Front Neurosci 9:113

Phillips KA, Sherwood CC (2008) Cortical development in brown capuchin monkeys: a structural MRI study. NeuroImage 43:657-664

Raichle ME (2015) The brain's default mode network. Annu Rev Neurosci 38:433-447

Ramasawmy R, Johnson SP, Roberts TA, Stuckey DJ, David AL, Pedley RB, Lythgoe MF, Siow B, Walker-Samuel S (2016) Monitoring the growth of an orthotopic tumour xenograft model: multi-modal imaging assessment with benchtop MRI (1T), high-field MRI (9.4T), ultrasound and bioluminescence. PLoS One 11(5):e0156162

Rilling JK (2006) Human and nonhuman primate brains: are they allometrically scaled versions of the same design? Evol Anthropol 15:65-77

Rilling JK (2014) Comparative primate neuroimaging: insights into human brain evolution. Trends Cogn Sci 18:46-55 
Rosenblum LA, Smith ELP, Altemus M, Scharf BA, Owens MJ, Nemeroff CB, Gorman JM, Coplan JD (2002) Differing concentrations of corticotropin-releasing factor and oxytocin in the cerebrospinal fluid of bonnet and pigtail macaques. Psychoneuroendocrinology 27:651-660

Russ BE, Leopold DA (2015) Functional MRI mapping of dynamic visual features during natural viewing in the macaque. NeuroImage 109:84-94

Scott JA, Grayson D, Fletcher E, Lee A, Bauman MD, Schumann CM, Buonocore MH, Amaral DG (2016) Longitudinal analysis of the developing rhesus monkey brain using magnetic resonance imaging: birth to adulthood. Brain Struct Funct 221:2847-2871

Seidlitz J, Sponheim C, Glen D, Ye FQ, Saleem KS, Leopold DA, Ungerleider L, Messinger A (2018) A population MRI brain template and analysis tools for the macaque. NeuroImage 170:121-131

Srihasam K, Sullivan K, Savage T, Livingstone MS (2010) Noninvasive functional MRI in alert monkeys. NeuroImage 51:267-273

Vanduffel W (2018) Long-term value memory in primates. PNAS 115:1956-1958

Wager TD, Yarkoni T (2012) Establishing homology between monkey and human brains. Nat Methods 9:237-239

Wisco JJ, Killiany RJ, Guttmann CR, Warfield SK, Moss MB, Rosene DL (2008) An MRI study of age-related white and gray matter volume changes in the rhesus monkey. Neurobiol Aging 29:1563-1575

Wittig RM, Crockford C, Deschner T, Langergraber KE, Ziegler TE, Zuberbuhler K (2014) Food sharing is linked to urinary oxytocin levels and bonding in related and unrelated wild chimpanzees. Proc Biol Sci 281(1778):20133096

Zitella LM, Xiao YZ, Teplitzky BA, Kastl DJ, Duchin Y, Baker KB, Vitek JL, Adriany G, Yacoub E, Harel N, Johnson MD (2015) In vivo 7T MRI of the non-human primate brainstem. PLoS One 10(5):e0127049

Open Access This chapter is licensed under the terms of the Creative Commons Attribution 4.0 International License (http://creativecommons.org/licenses/by/4.0/), which permits use, sharing, adaptation, distribution and reproduction in any medium or format, as long as you give appropriate credit to the original author(s) and the source, provide a link to the Creative Commons licence and indicate if changes were made.

The images or other third party material in this chapter are included in the chapter's Creative Commons licence, unless indicated otherwise in a credit line to the material. If material is not included in the chapter's Creative Commons licence and your intended use is not permitted by statutory regulation or exceeds the permitted use, you will need to obtain permission directly from the copyright holder. 